\title{
Saturated overburden scattering and the multiscatter frontier: Discovering dark matter at the Planck mass and beyond
}

\author{
Joseph Bramante, ${ }^{1,2}$ Benjamin Broerman, ${ }^{1}$ Rafael F. Lang, ${ }^{3}$ and Nirmal Raj ${ }^{4}$ \\ ${ }^{1}$ CPARC and Department of Physics, Engineering Physics, and Astronomy, Queen's University, \\ Kingston, Ontario K7L 2S8, Canada \\ ${ }^{2}$ Perimeter Institute for Theoretical Physics, Waterloo, Ontario N2L 2Y5, Canada \\ ${ }^{3}$ Department of Physics and Astronomy, Purdue University, West Lafayette, Indiana 47907, USA \\ ${ }^{4}$ Department of Physics, University of Notre Dame, Notre Dame, Indiana 46556, USA
}

(Received 30 March 2018; revised manuscript received 9 July 2018; published 11 October 2018)

\begin{abstract}
We show that underground experiments like LUX/LZ, PandaX-II, XENON, and PICO could discover dark matter up to the Planck mass and beyond, with new searches for dark matter that scatters multiple times in these detectors. This opens up significant discovery potential via reanalysis of existing and future data. We also identify a new effect that substantially enhances experimental sensitivity to large dark matter scattering cross sections: while passing through atmospheric or solid overburden, there is a maximum number of scatters that dark matter undergoes, determined by the total number of scattering sites it passes, such as nuclei and electrons. For dark matter heavier than $\sim 10^{15} \mathrm{GeV}$, this extends the reach of some published limits and future analyses to exclude large dark matter scattering cross sections, using detectors two kilometers underground.
\end{abstract}

DOI: $10.1103 /$ PhysRevD.98.083516

\section{INTRODUCTION}

While the presence of dark matter has been inferred from astrophysical and cosmological data, its nature remains enigmatic. Dark matter searches in the last few decades have sought out weakly interacting massive particles (WIMPs), which scatter at most once as they pass through underground detectors. We show how ongoing underground experiments could find dark matter that scatters multiple times as it travels through these detectors. We refer to these multiply interacting particles as MIMPs. We also show that a hitherto-neglected effect in dark matter studies, saturated overburden scattering (SOS), enhances published and prospective sensitivities to large scattering cross sections.

Dark matter more massive than the so-called unitarity limit of $\sim 100 \mathrm{TeV}$ arises naturally in grand unified theories that predict stable colored and electroweak states [1,2]. It has long been appreciated that they also arise in supersymmetric models [3], and can be produced out of equilibrium in the early Universe [4-8]. Depending on the reheating temperature, the primordial equation of state, and any substantial increases of entropy in the early Universe [9-13], superheavy dark matter could constitute

Published by the American Physical Society under the terms of the Creative Commons Attribution 4.0 International license. Further distribution of this work must maintain attribution to the author(s) and the published article's title, journal citation, and DOI. Funded by SCOAP ${ }^{3}$. the bulk of "missing mass" observed in galaxies. For low reheating temperatures, such dark matter could be a subdominant fraction of mass in the dark sector. Altogether, the discovery of superheavy particles may provide the first evidence for supersymmetry, grand unified theories, and new particle dynamics prior to big bang nucleosynthesis. This motivates the search for these particles in current and future experiments.

Here we investigate detection of such heavy, strongly interacting dark matter. In particular, in Sec. II, we detail the saturated overburden scattering effect, wherein dark matter scatters with every target along its path through the overburden. In Sec. III, we show the reach obtainable by existing direct detection experiments looking for multiply interacting dark matter, and evaluate its signatures. Section IV shows how, in much of the MIMP parameter space, the angle of entry into the detector can be used to validate signals, and to determine the mass, cross section, and local density of MIMPs with a single experiment.

\section{SATURATED OVERBURDEN SCATTERING}

On its path to a detector, dark matter may be slowed by scattering with the atmosphere, Earth overburden, and detector shielding. Usually, as the scattering cross section is increased, the kinetic energy of dark matter arriving at a detector is decreased, due to more frequent scatters en route. Because experiments require this kinetic energy to exceed some threshold to observe scattering, dark matter 

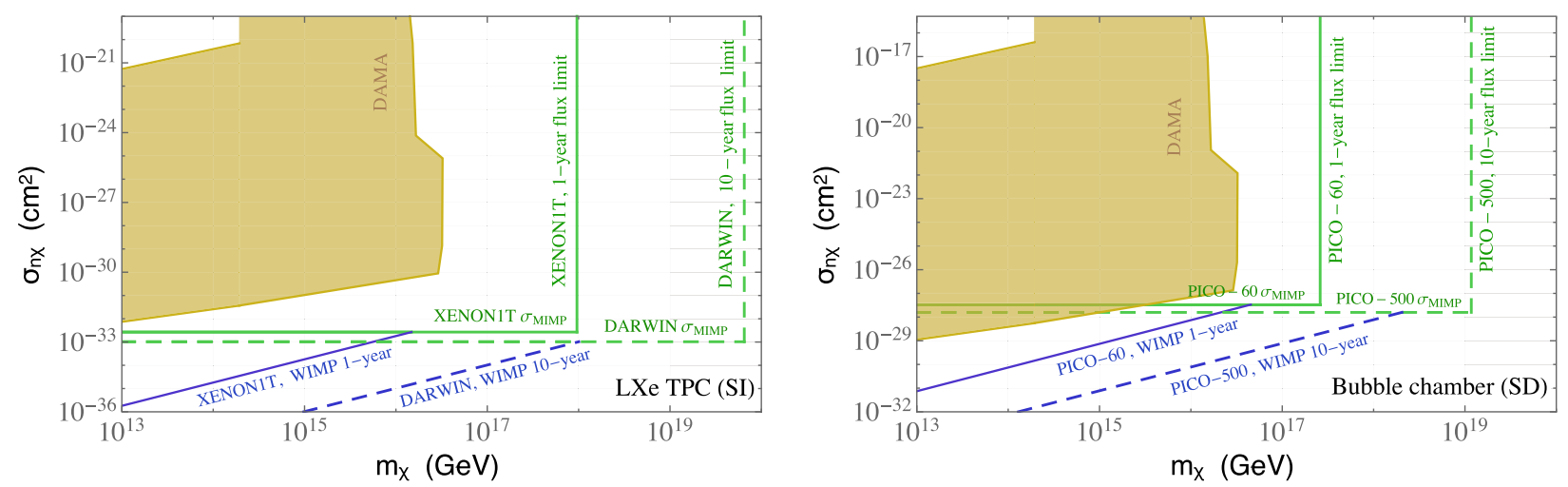

FIG. 1. Regions where dedicated multiscatter analyses are required for heavy dark matter are shown, given the examples of liquid xenon (for spin-independent scattering) or bubble chamber (spin-dependent) experiments. The (diagonal, blue) lines indicate the usual sensitivity from a zero-background single-scatter search. However, above the (horizontal, green) lines labeled $\sigma_{\text {MIMP }}$, more than $25 \%$ of the dark matter traversing the detector scatters more than once, requiring a dedicated multiscatter analysis to probe this parameter range. The ultimate high-mass reach is given by the (vertical, green) lines from the requirement that at least 2.3 dark matter particles traverse the detector for the stated effective detector area and exposure time. For detectors such as DARWIN and PICO-500, this limit can lie beyond the Planck mass $\sim 10^{19} \mathrm{GeV}$. These estimates are obtained assuming fiducial dimensions mentioned in Sec. III A and zero backgrounds as justified in Sec. III B; given the unique signatures of multiscattering dark matter and zero backgrounds, we expect future experimental sensitivities to lie close to these estimates. The blank region in the top left corner, where dark matter slowed down by the crust overburden does not trigger underground detectors, is beyond the reach of all the experiments mentioned here. For masses $\gtrsim 2 \times 10^{14} \mathrm{GeV}$, the crust overburden is insufficiently dense to slow down dark matter, and hence very high cross sections can be probed at underground detectors; this "saturated overburden scattering" is discussed in detail in Sec. II.

particles arriving too slowly become undetectable. This results in an upper limit on the cross-section sensitivity. However, above a certain cross section $\sigma_{\text {sos }}$, the dark matter scatters with every nucleus or electron along its path. Here we assume the dark matter particle is much smaller than nuclei or electrons, and that the maximum distance to a scattering site is set by the inverse momentum exchange. Increasing the cross section beyond $\sigma_{\text {sos }}$ does not further dampen the particle's kinetic energy.

We find that the optical depth $\tau_{\text {od }}$, i.e., the approximate total number of recoils ${ }^{1}$ from a single dark matter particle passing through an overburden of length $D$, scattering elastically with overburden nuclei or electrons is parametrically

$$
\tau_{\mathrm{od}}=\min \left[n \sigma D, n^{1 / 3} D\right],
$$

where $n$ is the number density and $n^{1 / 3}$ the inverse distance between nuclei or electrons, and $\sigma$ is the dark matternucleus or dark matter-electron scattering cross section. The min function accounts for the fact that dark matter scatters at most once with each of the $n^{1 / 3} D$ nuclei or electrons it passes. This treatment is valid so long as the typical momentum transfer is larger than the inverse

\footnotetext{
${ }^{1}$ Because of probabilistic variance in the total number of scatters one would not expect all dark matter particles to be stopped by the overburden, an effect that is important for $\tau_{\text {od }} \lesssim 100$ (as in [14]), but not for $\tau_{\text {od }} \approx 10^{10}$, as is the case when SOS is relevant.
}

distance between scattering sites, and applies specifically to superheavy dark matter in the Milky Way with velocity $\sim 0.001 c$ scattering elastically with nuclei in the Earth's crust. For nonperturbative interactions or scattering on targets other than earth-crust-density nuclei or speeds different from $0.001 \mathrm{c}$, Eq. (1) will need to be rescaled by an overall constant. From Eq. (1), the cross section for which overburden scattering is saturated is $\sigma_{\mathrm{sos}} \approx n^{-2 / 3}$ at the per-nuclear level. Note that this depends not on the length, but only the density, of the overburden.

Typical direct detection experiments are $\sim 1-2 \mathrm{~km}$ underground, where the Earth's crust constitutes the main overburden. We find that for spin-independent scattering the per-nucleon $\sigma_{\mathrm{sos}}=1.3 \times 10^{-20} \mathrm{~cm}^{2}$, assuming a crust density of $2.7 \mathrm{~g} / \mathrm{cm}^{3}$ and a crust composition as given in Sec. IV the most relevant SOS cross section arises from scattering with oxygen in the Earth's crust. Spin-dependent scatters occur mainly off ${ }^{27} \mathrm{Al}$, giving the per-nucleon $\sigma_{\text {sos }} \sim 10^{-18} \mathrm{~cm}^{2}$. Here we assume velocity-independent scattering and the usual relations to obtain per-nucleon cross sections [see Eq. (1) of [15]]. As noted in Fig. 1, the effect of saturating the overburden by scattering with all nuclei along the dark matter's path modifies prior DAMA bounds [16], extending the range of cross sections probed to arbitrarily large values for superheavy dark matter. The underground MIMP searches proposed in this document are sensitive to SI cross sections $\gtrsim 10^{-20} \mathrm{~cm}^{2}$ for MIMP masses in excess of $10^{15} \mathrm{GeV}$, and so can find MIMPs in the SOS regime. We leave the revisitation of other bounds vis a vis SOS to future work, which include those from the 
RRS balloon [17], the XQC calorimeter [14,18], CRESST-I $\mathrm{Al}_{2} \mathrm{O}_{3}$ experiments [19,20], as well as indirect bounds, e.g., from Earth heating [21]. We note that for a large enough nuclear scattering cross section, dark matter prohibits the cooling of interstellar molecular gas clouds in the Milky Way [22], which however only becomes relevant for $\sigma_{\mathrm{n} \chi} \gtrsim 10^{-10} \mathrm{~cm}^{2}\left(m_{\chi} /\left(10^{13} \mathrm{GeV}\right)\right)$ in the spin-independent case, where $m_{\chi}$ is the dark matter mass.

\section{DARK MATTER SCATTERING AT HIGH MULTIPLICITY}

For the high dark matter masses considered here, the probed cross sections are so large that particles may scatter with higher multiplicity than the customarily assumed single scatter. This is understood by examining the number of dark matter particles found by a detector,

$$
N_{\text {events }} \sim \Phi \min [\tau, 1],
$$

where $\Phi=\left(\rho_{\chi} / m_{\chi}\right) A_{\operatorname{det}} v_{\chi} t_{\exp }$ is the integrated flux of dark matter traversing the detector, $A_{\text {det }}$ the area of the detector, $\rho_{\chi}$ the average dark matter density, $v_{\chi}$ the average dark matter velocity, and $t_{\text {exp }}$ the exposure time. Dark matter undergoes a number of scatters roughly equal to the optical depth $\tau=n_{\text {det }} \sigma L_{\text {det }}$ where $L_{\text {det }}$ and $n_{\text {det }}$ are the detector length and number density of scattering sites.

Limits on $\sigma$ set by conventional searches are obtained in the single-scatter limit $\tau \ll 1$. Bounds on the scattering cross section $\left(\sigma_{\text {bound }}\right)$ weaken with increasing dark matter mass, $\sigma_{\text {bound }} \propto m_{\chi}^{-1}$. For high enough $m_{\chi}$ and hence $\sigma$, this scaling breaks down as $\tau \rightarrow 1$, since transiting particles begin to scatter multiple times in the detector. In a typical single-scatter search, such events will have been cut away in the data analysis. As we see, this breakdown occurs at a special point in $\left(m_{\chi}, \sigma_{\mathrm{T} \chi}\right)$ space where $\Phi \sim 1$ and $\tau \sim 1$. When all transiting dark matter scatters at least once $(\tau \gg 1)$, it is the detector area that determines MIMP sensitivity, unlike the single-scatter case, where it is determined by the volume.

For $\tau \gtrsim 1$, by setting $\Phi \sim 1$, we obtain the maximum mass to which an experiment is sensitive as $m_{\chi}^{\max } \sim \rho_{\chi} A_{\operatorname{det}} v_{\chi} t_{\exp }$ (as discussed also in [20,23]). Note that at the flux-limited masses we consider, using the average dark matter velocity of $\sim 10^{-3} \mathrm{c}$ to determine the flux gives nearly the same answer as integrating over a Boltzmann distribution. Typically, every generation of experiments increases $A_{\text {det }}$ as well as $t_{\text {exp }}$, thereby increasing $m_{\chi}^{\max }$. Thus for each new detector, there will be high-mass dark matter candidates that can only be uncovered with a multiscatter search.

\section{A. Prospects for multiscatter detection}

A number of prior studies have considered dark matter scattering multiple times while transiting detectors [14-20,23-28] and astronomical bodies [21,24,29-33].
Since our findings can be easily applied to any experiment with adequate sensitivity to identify multiple scatters, we simply consider two example technologies for dark matter detection: liquid xenon time projection chambers and bubble chambers. MIMPs could be found by analyzing data already collected at these experiments, such as the Xe detectors XENON1T [34], PandaX-II [35], and LUX [36], and the bubble chamber experiment PICO-60 [37] containing $\mathrm{C}_{3} \mathrm{~F}_{8}$. We also show the improvement in sensitivity at the future 50-ton detector DARWIN [38] and the future PICO upgrade, PICO-500 [39]. These experiments are poised to uncover MIMPs in regions that are orders of magnitude beyond past efforts such as DAMA (using NaI crystals) [16], and reanalyses of EDELWEISS (Ge) and CDMS (Ge, Si) [23], which were able to search for dark matter up to $m_{\chi} \lesssim 10^{16} \mathrm{GeV}$.

For convenience, we define a MIMP scattering threshold $\sigma_{\mathrm{MIMP}}=\left(L_{\mathrm{det}} n_{\mathrm{det}}\right)^{-1}$ as the cross section at which the Poisson expectation value for the number of recoils, per transit through the detector, from a single dark matter particle in a given detector is $\tau=1$. Given Poisson statistics, this happens when dark matter scatters at least twice during more than $25 \%$ of its detector transits.

Figure 1 shows the order-of-magnitude sensitivities achievable by MIMP searches. In practice, regions covered by searches where $\tau \gtrsim 1$ overlap due to Poisson fluctuations. Regions to the left of the solid green (dashed green) lines can be probed with 1-year (10-year) run time. This mass reach is determined by requiring that the integrated flux $\Phi$ is at least 2.3 events (corresponding to the 90\% confidence upper limit for zero observed events).

Single-scatter sensitivities $\sigma_{\text {single }}$ are shown for comparison, and are separated from MIMP regions by horizontal green lines at $\sigma_{\text {MIMP. }}$. These sensitivities are taken from Refs. [34-37,40] and rescaled using $L_{\text {det }}=\{96 \mathrm{~cm}, 250 \mathrm{~cm}$, $50 \mathrm{~cm}, 100 \mathrm{~cm}\}$ for $\{$ XENON1T, DARWIN, PICO-60, PICO-500\}. We assume densities of $\left\{2.94 \mathrm{~g} / \mathrm{cm}^{3}\right.$, $\left.1.36 \mathrm{~g} / \mathrm{cm}^{3}\right\}$ for $\left\{\mathrm{Xe}, \mathrm{C}_{3} \mathrm{~F}_{8}\right\}$ and $\left(J_{A},\left\langle S_{p}\right\rangle,\left\langle S_{n}\right\rangle\right)=$ $\left(\frac{1}{2}, 0.48,-0.01\right)$ for ${ }^{19} \mathrm{~F}[41]$. The relative behavior of these curves may be understood from their scalings with $L_{\text {det }}$, where for simplicity we take a spherical detector geometry. These scalings are $\sigma_{\mathrm{MIMP}} \propto L_{\text {det }}^{-1}, m_{\chi}^{\max } \propto L_{\text {det }}^{2} t_{\text {exp }}, \sigma_{\text {single }} \propto$ $\left(L_{\text {det }}^{3} t_{\text {exp }}\right)^{-1}$. Depending on their actual exposure times, detectors such as XENONnT [42], LZ [43], DARWIN and PICO-500 are capable of probing dark matter masses of the order $10^{19} \mathrm{GeV}$, i.e., up to and beyond the Planck mass.

\section{B. Signatures at high multiplicity}

A salient feature of superheavy MIMPs is that they will leave a mostly collinear track of nuclear recoils as they pass through a meter-scale detector. Comparing the maximum detector-frame scattering angle for MIMPs, given by the ratio of the scattering target to dark matter mass, $\sin \alpha_{\max }=m_{\mathrm{T}} / m_{\chi}$, with the maximum number of recoils 
in the detector $n_{\mathrm{det}}^{1 / 3} L_{\mathrm{det}}$, we find that in the limit $m_{\chi} \gg m_{\mathrm{T}}$ limit, the maximum total deflection angle of a dark matter particle is

$$
\begin{aligned}
\Omega_{\max } \lesssim & 1.7^{\circ}\left(\frac{m_{\mathrm{T}}}{100 \mathrm{GeV}}\right)\left(\frac{10^{13} \mathrm{GeV}}{m_{\chi}}\right) \\
& \times\left(\frac{L_{\mathrm{det}}}{100 \mathrm{~cm}}\right)\left(\frac{n_{\mathrm{det}}}{10^{22} \mathrm{~cm}^{3}}\right)^{1 / 3} .
\end{aligned}
$$

Thus, the nuclear recoils of a heavy transiting MIMP are typically collinear. We remark that, since solar and atmospheric neutrinos will not leave tracks in detectors, the "neutrino floor" $[44,45]$ is less of a concern for MIMP searches.

LUX, PandaX-II, and XENON1T are liquid xenon time projection chambers (TPCs) where, for each interaction, two signals are observed with photomultiplier tubes: an $\mathcal{O}(10 \mathrm{~ns})$ pulse (" $S_{1}$ ") from scintillation in the scattering target, followed by an $\mathcal{O}(\mu \mathrm{s})$ pulse (" $S_{2}$ ") from electrolumiscence of electrons that have drifted into the gas above the target liquid. The drift time is $\mathcal{O}(1 \mathrm{~ms})$, allowing for clear separation of $S_{1}$ and $S_{2}$. In comparison to these time scales, dark matter transits a $1 \mathrm{~m}$ detector length in $\sim 5 \mu \mathrm{s}$. The relative strength of $S_{1}$ vs $S_{2}$ helps distinguish dark matter-induced nuclear recoils from electronic recoils from $\beta$ and $\gamma$ radiation, which comprises the main background.

A MIMP transiting such a detector would produce multiple $S_{1}$ 's and $S_{2}$ 's, each characteristic of a nuclear recoil of relatively high energy in the range of 10 's of $\mathrm{keV}$. Whether the pulses would appear individually or merged is determined by the timing between successive scatters, typically the transit time divided by the number of recoils $\tau$. In the case of $S_{2}$ 's, distinguishing pulses will also depend on electron drift time. For $\tau \gtrsim 5(\tau \gtrsim 500)$ the $S_{2}\left(S_{1}\right)$ pulses merge into elongated pulses $S_{2}^{\prime}\left(S_{1}^{\prime}\right)$. There are thus three qualitatively distinct MIMP signatures: (1) a series of $S_{1}$ s followed by a series of $S_{2} \mathrm{~s}$, for $1 \lesssim \tau \lesssim 5$; (2) a series of $S_{1}$ s followed by a merged $S_{2}^{\prime}$, for $5 \lesssim \tau \lesssim 500$; and (3) an elongated $S_{1}^{\prime}$ followed by an $S_{2}^{\prime}$, for $\tau \gtrsim 500$, where the $S_{1}^{\prime}$ and $S_{2}^{\prime}$ overlap at least partially. Backgrounds to MIMP scattering will be exceedingly small. Signature (1) at small multiplicity can be mimicked by the pileup of individual single-scatter background events, which however happen predominantly at the surface of the detector and thus can be fiducialized. Another potential background in this regime is from fast decays such as the ${ }^{214} \mathrm{BiPo}$ coincidence which occurs in the ${ }^{222} \mathrm{Rn}$ decay chain [46,47], but will be of little concern since the alpha decay usually deposits much more energy than expected from MIMPs. At intermediate multiplicity, radiogenic neutrons might mimic the expected MIMP signature, but they do not usually travel at nonrelativistic speeds or scatter along a straight line. A background to elongated $S_{2}^{\prime}$ events comes from instrumental sources of drifting electrons [48,49], which will not usually conspire with multiple $S_{1}$ events to mimic the required MIMP signature. At large multiplicity, tracks from through-going muons will display much shorter $S_{1}$ pulses than expected from MIMPs, deposit much more energy than expected from dark matter, and can typically be vetoed by means of active shielding.

At PICO-60, the energy deposited by dark matter scattering off superheated $\mathrm{C}_{3} \mathrm{~F}_{8}$ nucleates a bubble, identified visually with stereo cameras and acoustically with piezoelectric transducers. A MIMP nucleates multiple bubbles during its $\sim 2.5 \mu$ s-long transit through the detector volume. The acoustics, sampled at a rate of $0.4 \mu \mathrm{s}^{-1}$, can identify at most 2.5/0.4 6 individual bubble nucleations, whereas the cameras could image up to 100 bubbles. With the background from radioactive neutrons limited [37], the visual position reconstruction makes PICO well suited for a MIMP search.

\section{DETERMINING THE MASS, CROSS SECTION, AND LOCAL DENSITY OF MIMPS}

The depletion of dark matter's kinetic energy after passing through a path $D$, along which lie nuclei with number densities $n_{i}$ and masses $m_{i}$, is

$$
\frac{E_{f}}{E_{i}}=\prod_{i}^{\text {nuclei }}\left(1-z \beta_{i}\right)^{\tau_{\mathrm{od}, i}},
$$

where $E_{i}$ and $E_{f}$ are respectively the initial and final dark matter kinetic energies and $z \beta_{i}=z 4 m_{i} m_{\chi} /\left(m_{\chi}+m_{i}\right)^{2}$ is the fraction of kinetic energy lost in each scatter, where $z$ is a kinematic factor set to $1 / 2$ for evenly distributed centerof-mass scattering angles [31], and the overburden optical depth for nuclear element $i$ is $\tau_{\mathrm{od}, i}$. To calculate this approximate upper bound on a detector's sensitivity to dark matter-nucleon scattering, we require that the final kinetic energy $E_{f}$ of a MIMP, which travels at typical galactic velocities of $10^{-3} c$, still has enough kinetic energy to deposit $1 \mathrm{keV}$ when recoiling against the target nucleus in an elastic collision.

To determine the number density of nuclei in the path of the dark matter as it transits either from above through the crust, or from below through the crust-mantle-core system, we model the interior of Earth using elemental abundances given in [50,51]. Figure 2 shows the spin-independent dark matter-nucleon cross sections above which the dark matter's kinetic energy is diminished so much that it becomes undetectable, for a path through $2 \mathrm{~km}$ of the crust or for the opposing radial path through $12756 \mathrm{~km}$ of Earth. Spindependent scattering occurs off $\mathrm{Al}, \mathrm{Na}, \mathrm{Ca}$, and ${ }^{29} \mathrm{Si}$, with nuclear spin expectation values $\left(\left\langle S_{p}\right\rangle,\left\langle S_{n}\right\rangle\right)$ given in [41].

With the overburden specified, it is possible to use the MIMP track direction as it transits the detector to determine the mass, cross section, and local density of MIMPs with a single experiment. We note that while typical directional dark matter detection effects rely on spatial anisotropy of 


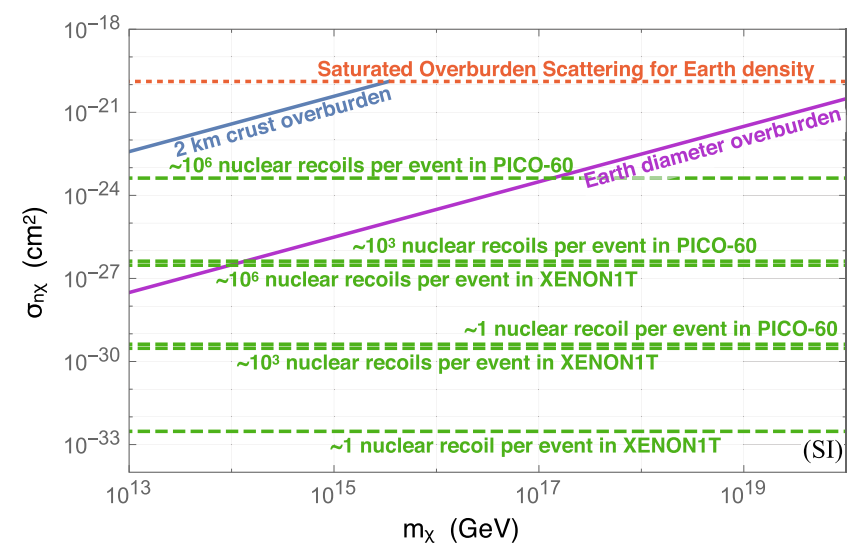

FIG. 2. The solid overburden lines indicate the boundaries for which the MIMP has lost all but $1 \mathrm{keV}$ of its kinetic energy propagating through either $2 \mathrm{~km}$ of Earth crust or through $12756 \mathrm{~km}$ of crust, mantle and core, where the region above and left of the Earth's crust line cannot be probed by underground experiments. In the parameter space between the overburden lines, it is possible to use the acceptance cone of the detector to pinpoint the mass, scattering cross section, and local density of MIMPs. Dashed lines indicate the typical number of recoils from a single dark matter particle passing through Xenon1T or PICO60. The SI saturated overburden scattering for Earth with density $2.7 \mathrm{~g} / \mathrm{cm}^{3}$ is indicated with a dotted line; note that the SD SOS cross section is $\sim 10^{-18} \mathrm{~cm}^{2}$.

incoming dark matter velocities (the dark matter velocity towards Earth is peaked in a direction pointing toward Cygnus), the effect we introduce here does not depend on dark matter velocity anisotropy, and arises simply because the overburden above and below a detector is different. For a separate effect that combines these modulations, see [52].

Our directional analysis is as follows: first, the number of target recoils from a single MIMP gives a measurement of the optical depth $\tau=n_{\mathrm{det}} \sigma_{T \chi} L_{\mathrm{det}}$, where the detector length $L_{\text {det }}$ and nuclear density $n_{\text {det }}$ are known. Thus the scattering cross section can be determined directly, and independently of the local dark matter density. Next, for parameter space between the two diagonal overburden lines in Fig. 2, some fraction of the MIMPs will not arrive at the detector: the arriving flux will have an angular dependence, since upwardgoing MIMPs will pass through more Earth overburden. More precisely, the MIMP mass and cross section uniquely determine the "angle of acceptance" at a direct detection experiment, defined as the angle between a vector pointing in the normal direction from the detector to the Earth's surface, and a vector pointing from the detector towards where the dark matter flux through the Earth is halved. This halving of flux occurs near an angle where all dark matter particles coming up through the Earth are blocked, which depends on dark matter's mass and velocity distribution; see Eq. (4). After appropriately modeling the dark matter velocity distribution and the Earth's interior, it would be possible, using Eq. (4), to pinpoint the dark matter mass.
(This is in contrast to usual single-scatter WIMP analyses where at high masses the spectral shape carries no information about the WIMP mass.) With the MIMP flux measured by the experiment $\left(\Phi=\left(\rho_{\chi} / m_{\chi}\right) A_{\operatorname{det}} v_{\chi} t_{\exp }\right)$ this is enough to determine the local MIMP density.

\section{OUTLOOK}

Grand unified, supersymmetric, and other theories motivate supermassive stable particles with per-nucleon cross sections in the $10^{-40}-10^{-20} \mathrm{~cm}^{2}$ range. We have demonstrated that multiply scattering massive particles, with masses in the $10^{10}-10^{20} \mathrm{GeV}$ range, require dedicated analyses, and may be discovered in data already collected by dark matter direct detection experiments, along with future experimental searches.

The new phenomenology presented in this article reveals many avenues for future research. Published limits on dark matter at large cross sections must be rederived after taking saturated overburden scattering into account. The sensitivity of dark matter detectors to leptophillic MIMPs scattering with target electrons must be explored. A directional analysis for validating MIMP signals incorporating a more detailed model of the Earth's interior can be undertaken. Incorporating correct detector geometry and the direction of drift in liquid xenon TPCs will result in diurnal modulation signatures. MIMP searches at detectors such as Borexino [53], SNO+ [54], JUNO [55], and MATHUSLA [56] should be investigated. Finally, the links between grand unified theories with stable states, the cosmological production of the same, and terrestrial searches for MIMPs should be sought out. Altogether, the search for dark matter in multiple-scatter events has the potential to revolutionize our understanding of fundamental physics and the early Universe.

\section{ACKNOWLEDGMENTS}

We thank Jimmy Bramante, Mark Chen, Ken Clark, Adrienne Erickcek, Jonathan Feng, Gilles Gerbier, Guillaume Giroux, Bradley Kavanagh, Graham Kribs, Jason Kumar, Adam Martin, Sam McDermott, Tony Noble, Maxim Pospelov, Alan Robinson, Jessie Shelton, Louis Strigari, Aaron Vincent, and Alex Wright for useful conversations. We also thank anonymous referees for numerous helpful comments and clarifications. We thank the Perimeter Institute for Theoretical Physics, where this work was initiated. Research at Perimeter Institute is supported by the Government of Canada through Industry Canada and by the Province of Ontario through the Ministry of Economic Development \& Innovation. J. B. and B. B. acknowledge the support of the Natural Sciences and Engineering Research Council of Canada. J. B. thanks the Aspen Center for Physics, which is supported by National Science Foundation (NSF) Grant No. PHY-1066293. The NSF also supports R. F. L. through Grant No. PHY-1719271 and N. R. through Grants No. PHY-1417118 and No. PHY-1520966. 
[1] S. Burdin, M. Fairbairn, P. Mermod, D. Milstead, J. Pinfold, T. Sloan, and W. Taylor, Noncollider searches for stable massive particles, Phys. Rep. 582, 1 (2015).

[2] K. Griest and M. Kamionkowski, Unitarity Limits on the Mass and Radius of Dark Matter Particles, Phys. Rev. Lett. 64, 615 (1990).

[3] S. Raby, Gauge mediated SUSY breaking at an intermediate scale, Phys. Rev. D 56, 2852 (1997).

[4] D. J. H. Chung, E. W. Kolb, and A. Riotto, Superheavy dark matter, Phys. Rev. D 59, 023501 (1998).

[5] V. Kuzmin and I. Tkachev, Matter creation via vacuum fluctuations in the early Universe and observed ultrahighenergy cosmic ray events, Phys. Rev. D 59, 123006 (1999).

[6] K. Harigaya, M. Kawasaki, K. Mukaida, and M. Yamada, Dark matter production in late time reheating, Phys. Rev. D 89, 083532 (2014).

[7] K. Harigaya, T. Lin, and H. K. Lou, GUTzilla dark matter, J. High Energy Phys. 09 (2016) 014.

[8] E. W. Kolb and A. J. Long, Superheavy dark matter through Higgs portal operators, Phys. Rev. D 96, 103540 (2017).

[9] J. Bramante and J. Unwin, Superheavy thermal dark matter and primordial asymmetries, J. High Energy Phys. 02 (2017) 119.

[10] G. Gelmini, P. Gondolo, A. Soldatenko, and C. E. Yaguna, The effect of a late decaying scalar on the neutralino relic density, Phys. Rev. D 74, 083514 (2006).

[11] G. Kane, J. Shao, S. Watson, and H.-B. Yu, The baryon-dark matter ratio via moduli decay after Affleck-Dine baryogenesis, J. Cosmol. Astropart. Phys. 11 (2011) 012.

[12] L. Randall, J. Scholtz, and J. Unwin, Flooded dark matter and S level rise, J. High Energy Phys. 03 (2016) 011.

[13] H. Davoudiasl, D. Hooper, and S. D. McDermott, Inflatable Dark Matter, Phys. Rev. Lett. 116, 031303 (2016).

[14] M. S. Mahdawi and G. R. Farrar, Closing the window on $\sim \mathrm{GeV}$ dark matter, J. Cosmol. Astropart. Phys. 12 (2017) 004.

[15] D. Hooper and S. D. McDermott, Robust constraints and novel gamma-ray signatures of dark matter that interacts strongly with nucleons, Phys. Rev. D 97, 115006 (2018).

[16] R. Bernabei et al., Extended Limits on Neutral Strongly Interacting Massive Particles and Nuclearites from NaI(Tl) Scintillators, Phys. Rev. Lett. 83, 4918 (1999).

[17] J. Rich, R. Rocchia, and M. Spiro, A search for strongly interacting dark matter, Phys. Lett. B 194, 173 (1987).

[18] A. L. Erickcek, P. J. Steinhardt, D. McCammon, and P. C. McGuire, Constraints on the interactions between dark matter and baryons from the $\mathrm{x}$-ray quantum calorimetry experiment, Phys. Rev. D 76, 042007 (2007).

[19] J. H. Davis, Probing Sub-GeV Mass Strongly Interacting Dark Matter with a Low-Threshold Surface Experiment, Phys. Rev. Lett. 119, 211302 (2017).

[20] B. J. Kavanagh, Earth scattering of superheavy dark matter: Updated constraints from detectors old and new, Phys. Rev. D 97, 123013 (2018).

[21] G. D. Mack, J. F. Beacom, and G. Bertone, Towards closing the window on strongly interacting dark matter: Farreaching constraints from Earth's heat Flow, Phys. Rev. D 76, 043523 (2007).

[22] R. S. Chivukula, A. G. Cohen, S. Dimopoulos, and T. P. Walker, Bounds on Halo Particle Interactions From Interstellar Calorimetry, Phys. Rev. Lett. 65, 957 (1990).
[23] I. F. M. Albuquerque and L. Baudis, Direct Detection Constraints on Superheavy Dark Matter, Phys. Rev. Lett. 90, 221301 (2003); Erratum, Phys. Rev. Lett., 91, 229903 (E) (2003).

[24] G. D. Starkman, A. Gould, R. Esmailzadeh, and S. Dimopoulos, Opening the window on strongly interacting dark matter, Phys. Rev. D 41, 3594 (1990).

[25] P. C. McGuire, Low background balloon borne direct search for ionizing massive particles as a component of the dark galactic halo matter, Ph. D. thesis, Arizona University, 1994.

[26] B. D. Wandelt, R. Dave, G. R. Farrar, P. C. McGuire, D. N. Spergel, and P. J. Steinhardt, in Self-interacting dark matter, edited by D. B. Cline, Sources and Detection of Dark Matter and Dark Energy in the Universe (Springer, Berlin, Heidelberg, 2000), pp. 263-274.

[27] G. Zaharijas and G. R. Farrar, A window in the dark matter exclusion limits, Phys. Rev. D 72, 083502 (2005).

[28] C. Kouvaris and I. M. Shoemaker, Daily modulation as a smoking gun of dark matter with significant stopping rate, Phys. Rev. D 90, 095011 (2014).

[29] R. Foot and S. Vagnozzi, Diurnal modulation signal from dissipative hidden sector dark matter, Phys. Lett. B 748, 61 (2015).

[30] B. J. Kavanagh, R. Catena, and C. Kouvaris, Signatures of Earth scattering in the direct detection of dark matter, J. Cosmol. Astropart. Phys. 01 (2017) 012.

[31] J. Bramante, A. Delgado, and A. Martin, Multiscatter stellar capture of dark matter, Phys. Rev. D 96, 063002 (2017).

[32] T. Emken and C. Kouvaris, DaMaSCUS: The impact of underground scatterings on direct detection of light dark matter, J. Cosmol. Astropart. Phys. 10 (2017) 031.

[33] T. Emken and C. Kouvaris, How blind are underground and surface detectors to strongly interacting dark matter? Phys. Rev. D 97, 115047 (2018).

[34] E. Aprile et al. (XENON), First Dark Matter Search Results from the XENON1T Experiment, Phys. Rev. Lett. 119, 181301 (2017).

[35] X. Cui et al. (PandaX-II), Dark Matter Results From 54-Ton-Day Exposure of PandaX-II Experiment, Phys. Rev. Lett. 119, 181302 (2017).

[36] D. S. Akerib et al. (LUX), Results from a Search for Dark Matter in the Complete LUX Exposure, Phys. Rev. Lett. 118, 021303 (2017).

[37] C. Amole et al. (PICO), Dark Matter Search Results from the PICO-60 $\mathrm{C}_{3} \mathrm{~F}_{8}$ Bubble Chamber, Phys. Rev. Lett. 118, 251301 (2017).

[38] J. Aalbers et al. (DARWIN), DARWIN: Towards the ultimate dark matter detector, J. Cosmol. Astropart. Phys. 11 (2016) 017.

[39] E. Vázquez-Jáuregui for the PICO collaboration, Pico500L: Simulations for a 500L bubble chamber for dark matter search, https://indico.cern.ch/event/606690/ contributions/2591726/, 2017.

[40] D. S. Akerib et al. (LUX), Limits on Spin-Dependent WIMP-Nucleon Cross Section Obtained from the Complete LUX Exposure, Phys. Rev. Lett. 118, 251302 (2017).

[41] V. A. Bednyakov and F. Simkovic, Nuclear spin structure in dark matter search: The zero momentum transfer limit, Fiz. Elem. Chastits At. Yadra 36, 257 (2005) [Phys. Part. Nucl. 36, 131 (2005)]. 
[42] E. Aprile et al. (XENON), Physics reach of the XENON1T dark matter experiment, J. Cosmol. Astropart. Phys. 04 (2016) 027.

[43] D. S. Akerib et al. (LUX-ZEPLIN), Projected WIMP sensitivity of the LUX-ZEPLIN (LZ) dark matter experiment, arXiv:1802.06039.

[44] J. Billard, L. Strigari, and E. Figueroa-Feliciano, Implication of neutrino backgrounds on the reach of next generation dark matter direct detection experiments, Phys. Rev. D 89, 023524 (2014).

[45] F. Ruppin, J. Billard, E. Figueroa-Feliciano, and L. Strigari, Complementarity of dark matter detectors in light of the neutrino background, Phys. Rev. D 90, 083510 (2014).

[46] E. Aprile et al. (XENON), Intrinsic backgrounds from $\mathrm{Rn}$ and $\mathrm{Kr}$ in the XENON100 experiment, Eur. Phys. J. C 78, 132 (2018).

[47] S. Li, X. Chen, X. Cui, C. Fu, X. Ji, Q. Lin, J. Liu, X. Liu, A. Tan, X. Wang, M. Xiao, and P. Xie, Krypton and radon background in the pandax-i dark matter experiment, J. Instrum. 12, T02002 (2017).

[48] P. Sorensen, Electron train backgrounds in liquid xenon dark matter search detectors are indeed due to thermalization and trapping, arXiv:1702.04805.
[49] P. Sorensen and K. Kamdin, Two distinct components of the delayed single electron noise in liquid xenon emission detectors, J. Instrum. 13, P02032 (2018).

[50] F. W. Clarke and H. S. Washington, The composition of the Earth's crust (1924).

[51] J. W. Morgan and E. Anders, Chemical composition of Earth, Venus, and Mercury, Proc. Natl. Acad. Sci. U.S.A. 77, 6973 (1980).

[52] J. I. Collar and F. T. Avignone, III, The effect of elastic scattering in the Earth on cold dark matter experiments, Phys. Rev. D 47, 5238 (1993).

[53] G. Alimonti et al. (Borexino), Science and technology of BOREXINO: A real time detector for low-energy solar neutrinos, Astropart. Phys. 16, 205 (2002).

[54] S. Andringa et al. (SNO+), Current status and future prospects of the $\mathrm{SNO}+$ Experiment, Adv. High Energy Phys. 2016, 6194250 (2016).

[55] Y.-F. Li, Overview of the Jiangmen Underground Neutrino Observatory (JUNO), in Proceedings, 33rd International Symposium on Physics in Collision (PIC 2013), Beijing, China, 2013, http://inspirehep.net/record/1282440.[Int. J. Mod. Phys. Conf. Ser. 31, 1460300 (2014)].

[56] J. P. Chou, D. Curtin, and H. J. Lubatti, New detectors to explore the lifetime frontier, Phys. Lett. B 767, 29 (2017). 